\title{
LES STROMATOLITES GLOBULEUX DE LA FAZENDA SERRA VIRGEM (MINAS GERAIS)
}

\author{
J.P. and J.O. CASSEDANNE*
}

\begin{abstract}
Near Curvelo (Minas Gerais), a new stromatolitic occurrence in oolitic limestone belonging to Lagoa do Jacaré Member, is described.

Lithologic section and microscopic facies of rocks surrounding the fossiliferous bed are given in order to definite the environmental conditions of sedimentation.

The stromatolites are "cumulate separate" type, in a sparce biostrome. They are pillowshaped, with variable thickness and steep borders. Microstructure is of single film type.

The wall-rock is a detritical oolitic limestone with slabs of hardened rock and elongated stromatolite fragments.

Comparison with similar forms described in Siberia suggests an upper Riphean age for the fossiliferous deposit.
\end{abstract}

RESUMO Uma nova jazida de estromatólitos é descritados calcários oolíticos do Membro Lagoa do Jacaré, perto de Curvelo (Minas Gerais).

O perfil litológico e as microfácies das rochas que encaixam o horizonte fossilifero são descritos, visando definir as condiçōes de sedimentação.

Os estromatólitos, formando um biostromo pouco denso, apresentam a forma de uma almofada, de espessura variável, cujos bordos são algumas vezes abruptos. A microestrutura é simples, do tipo filme.

A rocha encaixante é um calcário oolítico grosseiro, com fragmentos de rocha endurecida e placas alongadas de estromatólitos.

Comparação com formas descritas, principalmente na Sibéria, sugerem uma idade Rifeano Superior para os fósseis.

INTRODUCTION De nombreux gites de stromatolites columnaires, le plus souvent localisés dans des ensembles plus ou moins dolomitiques, rapportés à la Formation Sete Lagoas, sont connus dans le Groupe Bambui (Marchese, 1974).

Nous nous proposons de décrire ci-dessous un nouveau gisement, inclus dans une lentille de calcaire oolithique intercalé dans des siltites (Membre Lagoa do Jacaré). Indépendemment de leur environnement uniquement calcaire, ces stromatolites se caractérisent par une forme globuleuse qui ne parait pas encore avoir été décrite au Brésil.

Localisation. Moyens d'acces Les stromatolites, objet de la présente étude, proviennent d'un groupe d'excavations également connu sous le nom de "Pedreira Alcindo Vieira", situé à quelques centaines de mètres de la route Belo HorizonteBrasilia. Cette carrière, ouverte dans la Fazenda Serra Virgem (Municipe de Curvelo, Minas Gerais), est facilement accessible par une bonne piste de $0,7 \mathrm{~km}$ qui s'embranche en face du chemin de Pompéu, au kilomètre 577 de la route Belo Horizonte-Brasilia.

\footnotetext{
* Instituto de Geociências - C.C.M.N. \& C.N.Pq Universidade Federal do Rio de Janeiro - Ilha do
} Fundão - Cidade Universitária 
Contexte geologique Les carrières, ouvertes dans le but de fournir le ballast nécessaire à l'encaillassement et aux ouvrages d'art de la grande route voisine, sont actuellement abandonnées. Elles ont entaillé une petite élévation calcaire arrondie s'élevant au-dessus d'une série de collines mollement creusées dans les siltites.

Le calcaire de ces carrières a été choisi comme type du Membre Lagoa do Jacaré par Branco \& da Costa (1961). Il repose sur les siltites du Membre Serra de Santa Helena, base de la Formation Rio Paraopeba. Les siltites du Membre Três Marias le recouvrent.

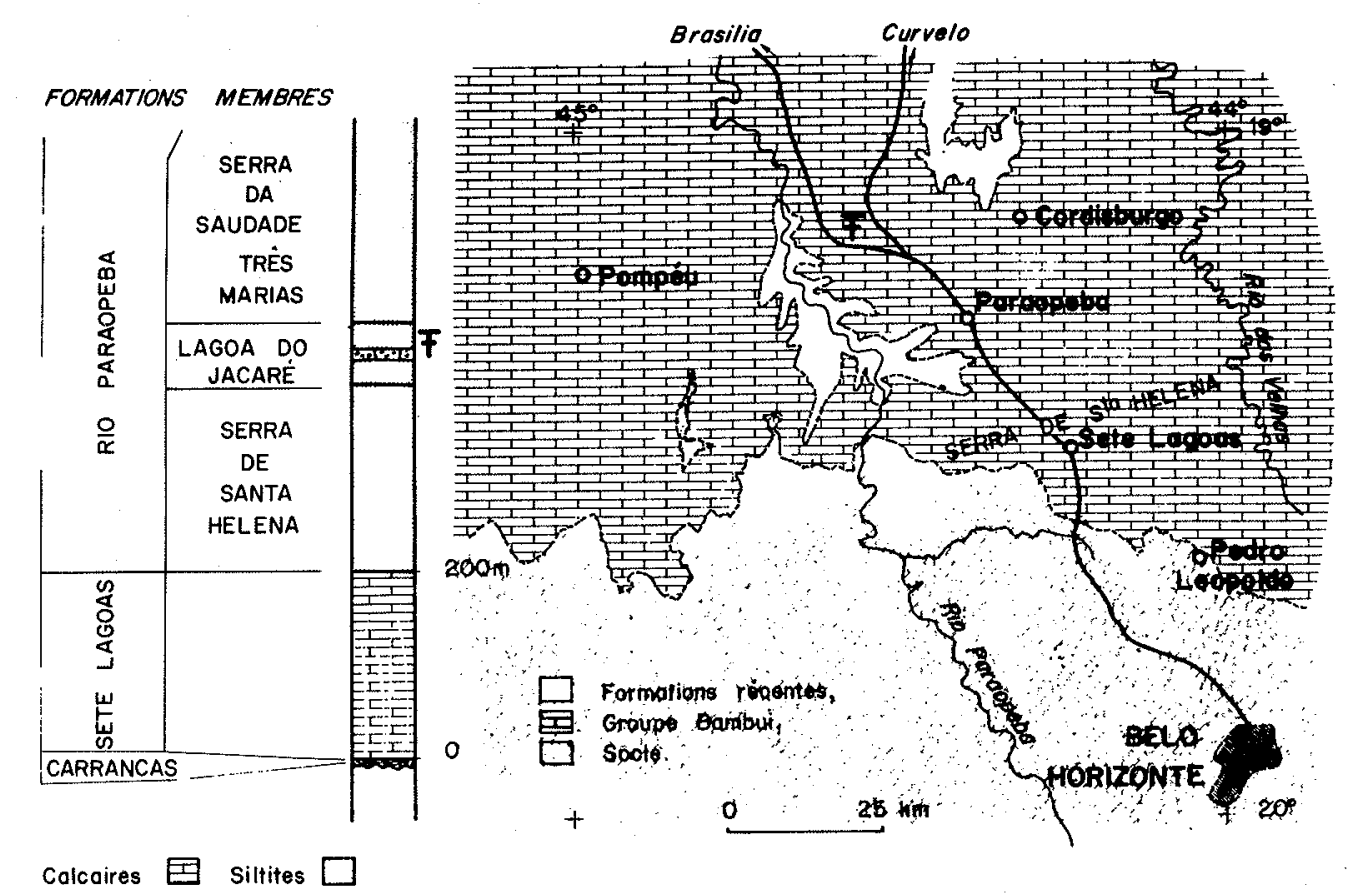

Figure 1 - Localisation du gîte fossilifère et situation de l'horizon stromatolițque dans la colonne lithologique du Groupe Bambui

Rappelons que le Membre Lagoa do Jacaré a été élevé au rang de Formation par Barbosa (1965) alors qu'il ne constitue qu'un faciès de la Formation Paraopeba de Braun (1968).

On se reportera aux travaux des auteurs précités pour une description pétrographique et tectonique des diverses formations précédentes.

Afín de préciser l'ambiance de sédimentation des calcaires constituant la colline de Serra Virgem, nous décrirons la coupe stratigraphique de cette élévation, avant d'aborder l'étude des stromatolites.

Coupe stratigraphique de la colline de Serra Virgem. Du pied de la colline à son sommet une série d'affleurements et d'excavations superposées correspondant à la carriére principale, permettent de donner la coupe suivante, incluant tous les calcaires du faciès Lagoa do Jacaré. Les numéros entre parenthèses renvoient à la Figure 2 et indiquent les microfaciès figurés. 


\section{PROFIL LITHOLOGIQUE ET MICROFACIES}

\section{DE LA COLLINE DE SERRA VIRGEM}

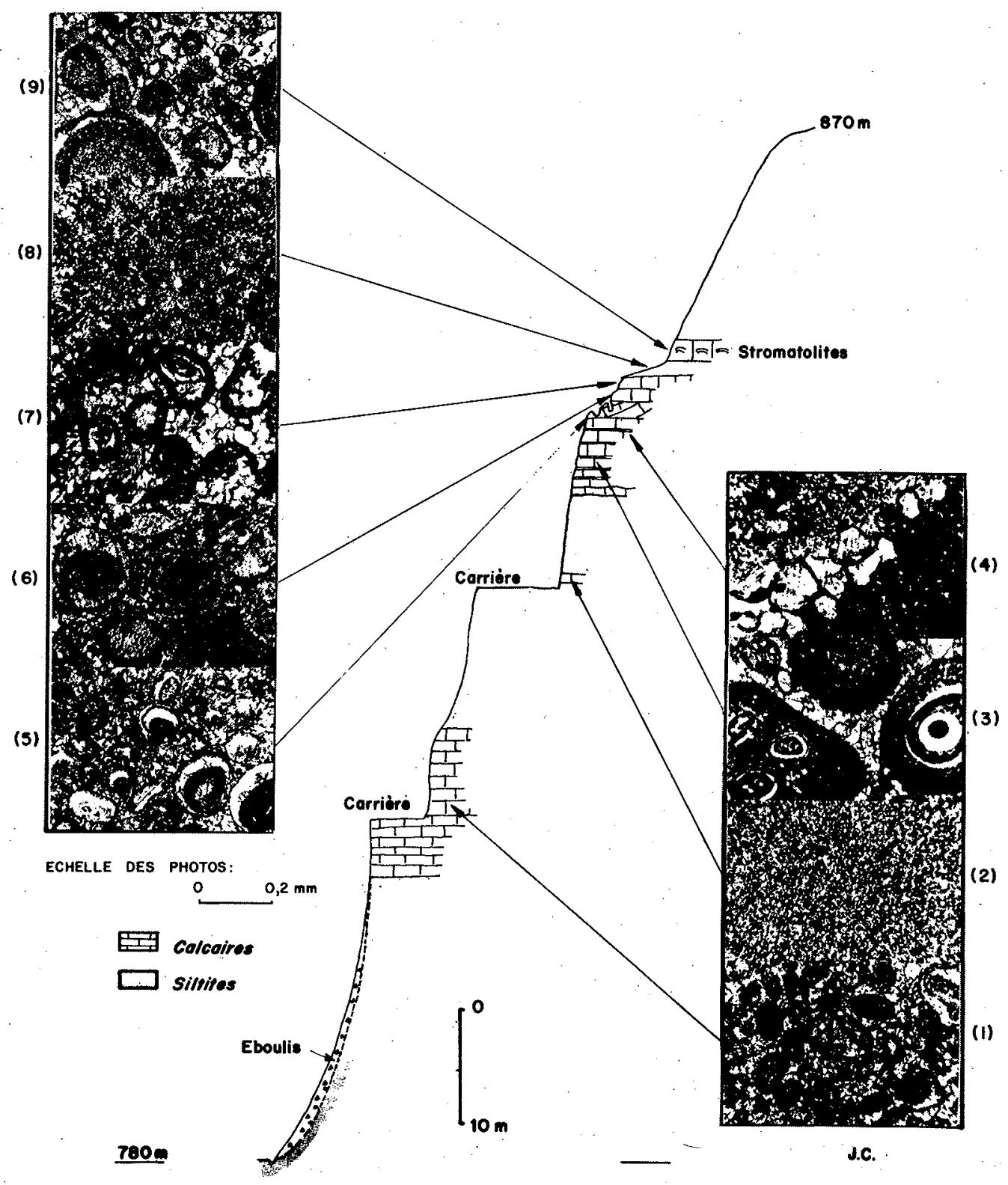

Figure 2 - Profil lithologique de la colline de Serra Virgem et principaux microfaciès. Les numéros accompagnant les photographies de ces derniers correspondent aux descriptions du texte. Lames minces toutes reproduites à la même échelle 
SILTITES SCHISTEUX On les trouve en fragments anguleux typiques dans le lit d'un ruisseau coulant au pied de l'élévation. Ils montent, recouverts par les éboulis de pente, jusqu'au voisinage de la cote 805 (ex faciès Serra de Santa Helena ou Serra da Gineta).

CALCAIRES INFERIEURS Ils débutent par des bancs bien lités à minces interlits argileux, dont le contact avec les siltites sous-jacents est masqué. Au-dessus repose un calcaire plus grossier à lits oolithiques et stratification oblique où on observe des lentilles irrégulières de conglomérat intraformationnel. Au pied des anciennes trémies la surface de la roche, grise, est très finement ondulée et présente de petites oolithes en relief. Sur la cassure brillent des cristaux de calcite.

Au microscope, la roche se montre recristallisée en fine mosaïque. Les oolithes sont irrégulières, à peine plus foncées que le ciment, où pyrite et quartz sont rares (1). Leur structure est en partie effacée par la recristallisation : certaines sont composées, d'autres ont un noyau de calcite. Des plages de calcite largement cristallisée envahissent la roche où des stylolithes sont soulignés par des impuretés brunes.

DES PHYLLITES NON CALCAIRES, gris verdâtre, d'aspect satiné, à peine métamorphiques et finnement rubannées surmontent les calcaires. Riches en pyrite et limonite, elles se débitent en plaquettes irrégulières d'épaisseur multicentimétrique. On y remarque de grands ripple-marks sur les joints de stratification.

Deux mètres de calcaire compact en lits multidécimétriques s'y intercalent localement (Est et entrée de la carrière). Il est clair, homogène et sa cassure est conchoïdale. C'est une micrite aux trainées brunâtres limitées, présențant des paillettes de mica dispersées sans orientation déterminée.

De nombreuses cassures verticales $\mathrm{N} 100^{\circ} \mathrm{E}$, recoupant les phyllites, ont été élargies par l'érosion. De la calcite beige ou orangée, en draperies largement cristallisées, les tapisse.

CALCAIRES SUPERIEURS C'est un ensemble d'une huitaine de mètres, reposant sur les phyllites par un banc de $0,8 \mathrm{~m}$ aux grands ripple-marks, qui constituait la sole de la carrière primitive. Au-dessus, des calcaires gris noirâtre massifs, à interlits de phyllites et lentilles de conglomérat intraformationnel, deviennent bien stratifiés vers le haut. Les deux mètres du sommet, très oolithiques et karstifiés, présentent une forte stratification oblique (3). La plupart des roches précédentes prend une patine blanchâtre par oxydation de la matière organique. Les filonnets de calcite noire y sont fréquents ainsi que la limonite.

Ces calcaires, déposés sous un régime hydraulique beaucoup plus violent que celui des phyllites, offrent de belles figures de sédimentation agitée.

Le calcaire du niveau le plus bas (2), gris noirâtre foncé, a une cassure esquilleuse à subconchoïdale. C'est une micrite où se notent seulement quelques noyaux irréguliers plus grossiers.

Le poudingue intraformationnel (4), calcaire, est formé de galets ovoïdes beige, gris clair à verdâtre, argileux, noyés dans un ciment plus foncé à plages oolithiques et grands noyaux de calcite noire. Au microscope, les galets, homogènes, tachés de matière organique et/ou de limonite sur leurs bords, présentent un contact diffus avec le ciment qui les a moulé à l'état pâteux, en pénétrant à leur périphérie. Les oolithes du ciment, microsparite, sont un peu plus foncées que celui-ci qui, localement est une sparite. 
Un conglomérat identique à galets mous a été décrit le long de la route de Montes Claros à Januaria, dans le même Etat; un autre affleure près de l'indice de plomb de Cascavel (Minas Gerais). Rappelons qu'une compilation bibliographique confirme que ces galets sont presque toujours d'origine marine côtière et attestent une phase d'émersion (Cassedanne, 1966). Un réseau de fentes de dessication qui était visible sur la sole de l'ancienne carrière confirme celle-ci.

Le calcaire oolithique supérieur (5) présente en affleurements de petites oolithes gris blanc sur un fond plus foncé. Sur la cassure celles-ci se montrent en trainées sinueuses, en nids ou irrégulièrement dispersées. Au microscope la roche se caractérise par la petite taille de ses oolithes souvent extrêmement recristallisées ou en couches épaisses non concentriques. Souvent allongées, elles sont localement presque jointies ou orientées suivant la même direction, leur noyau étant excentré, comme si un classement s'était produit pendant la sédimentation. Les débris de roche, allongés, sont fréquents, comme les stylolithes.

DEUX METRES DE CALCAIRE OOLITHIQUE gris foncé reposent sur les calcaires précédents. Ce sont des lits centi à décimétriques séparés par des phyllites analogues à celles de la carrière. Sur la cassure, des facettes spathiques sont mêlées à des oolithes gris souris noyées dans un ciment à peine plus foncé. Au microscope, dans un ciment spathique, les oolithes sont remplacées par un seul cristal de calcite ou plus ou moins effacées par la diagenèse. Quelques autres ont des couches parallèles minces ou plus épaisses séparées par de la calcite en large mosaïque; certaines sont excentrées, d'autres composées (6).

1,5 METRE DE CALCAIRE SPATHIQUE, gris foncé, homogène, aux ondulations irrégulières, surmonte le calcaire précédent; quelques lits en sont soulignés par de minces couches argileuses beige clair. En affleurement les oolithes sont creusées en minuscules cupules, alors que sur les plans de fracture elles présentent des facettes de clivage brillantes. Au microscope, la recristallisation a en partie effacée la structure. Les oolithes de calcite secondaire, moulant quelquefois un noyau de quartz, sont toujours assez petites, un peu plus foncées que le ciment. Certaines sont composées, excentrées, de taille et forme irrégulières (7).

DEUX METRES DE CALCAIRE ARGILEUX forment replat. En plaquettes, il est homogène, gris beige, et donne par altération un sol argileux. C'est une micrite maculée par beaucoup d'argile où la pyrite en cristaux de taille variable est abondante (8).

LE BANC FOSSILIFERE, puissant de 3 mètres, forme le sommet de l'excavation. C'est un calcaire oolithique gris foncé à patine blanchatre, très grossier, enveloppant les structures organiques. Sur la cassure des oolithes gris souris foncé sont en relief ou, cassées, brillent; entre celles-ci, de plus petites et des gravelles plus claires, sont irrégulièrement mêlées à des fragments de roche. Des taches de calcite noire existent localement. Par dissolution les oolithes laissent de petites cupules sur les affleurements, où les débris calcaires restent en relief sur un fond un peu plus foncé (9).

La description microscopique et l'analyse de cette roche seront données ultérieurement.

SILTITES QUARTZEUX BEIGES Ils forment le sommet de l'élévation, audessus du banc fossilifère, jusqu'à la cote 870 (ex faciès Três Marias). Pratiquement dépourvus d'affleurements, ils donnent une topographie molle. 
Du point de vue tectonique, tout l'ensemble sédimentaire plonge très légèrement vers l'Est

Second groupe de carrières Au SW des carrières précédentes, plusieurs autres excavations ont été ouvertes à la cote 865 . Elles sont accessibles par une piste carrossable tracée sur des phyllites à fort pendage Ouest dû à un plissotement local.

Les calcaires correspondant aux calcaires supérieurs des autres carrières sont massifs, gris foncé, bien lités, mais un peu plus homogènes et d'un grain à peine plus fin. Pyrite et interlits phylliteux y sont également un peu moins fréquents.

A la cote 875, un joint creusé par l'érosion sépare des calcaires subhorizontaux, grossiers, assez bien lités, d'un tapis sur lequel ont crû des stromatolites. Ceux-ci seront décrits avec ceux des autres carrières, étant identiques.

Description generale des stromatolites 1 - AFFLEUREMENTS Les organismes sont constitués par une alternance de bandes claires et foncées, de dimensions variables, ondulées, s'anastomosant fréquemment et incluant très localement des pellicules lenticulaires d'argile.

Sections verticales. Elles sont dûes à l'exploitation des carrières ou à l'érosion, dégageant des joints verticaux et mettant en relief les structures, ce qui permet de visualiser leur coupe.

Les stromatolites se développent sur une base rappelant un tapis d'algues, audessus d'un joint de stratification, leur courbure s'accentue ensuite, surtout latéra-

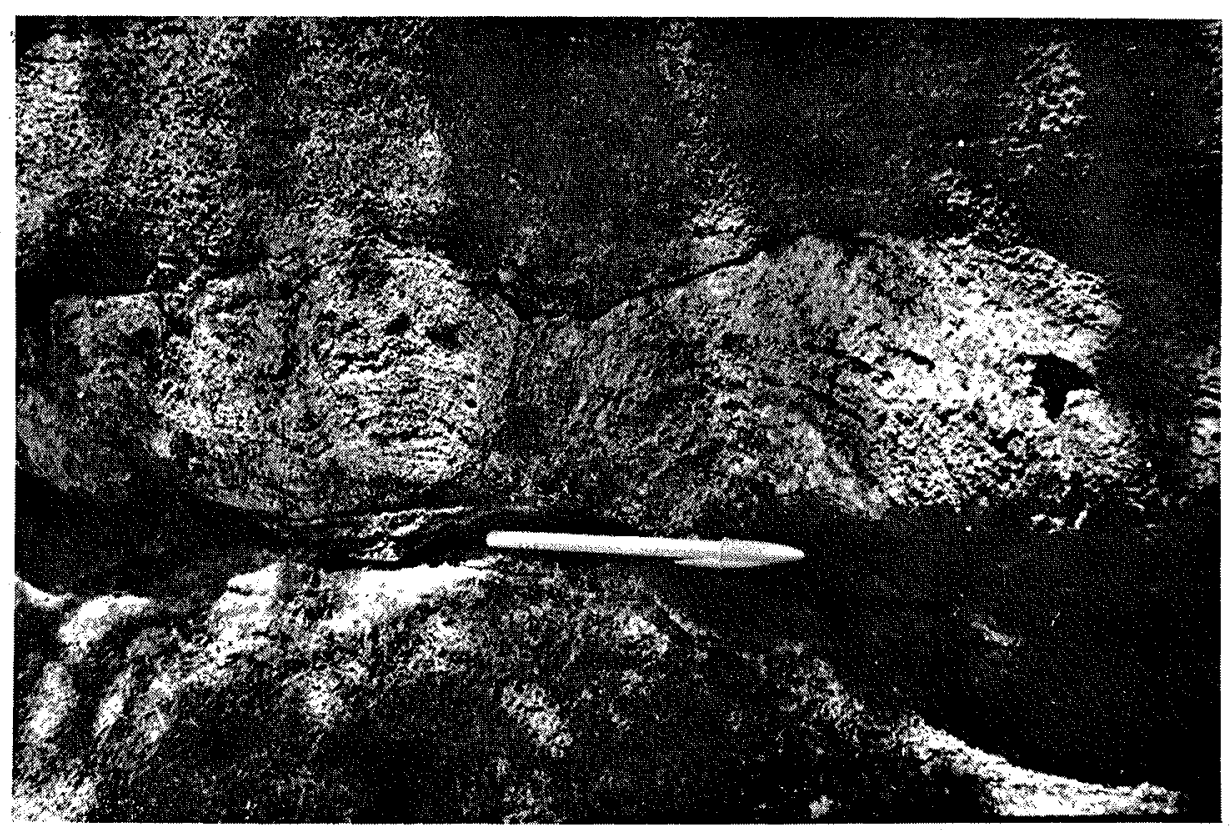

Photo 1 - Affleurement de stromatolttes sur une paroi verticale de la carrière SW 
lement au fur et à mesure de leur croissance, sans toutefois dépasser la forme d'une large calotte. Les lamelles débordent quelquefois leur base et peuvent devenir verticales (structure en coffre). Leur contact est alors très abrupt avec la roche encaissante qui présence des invaginations dans la structure organique.

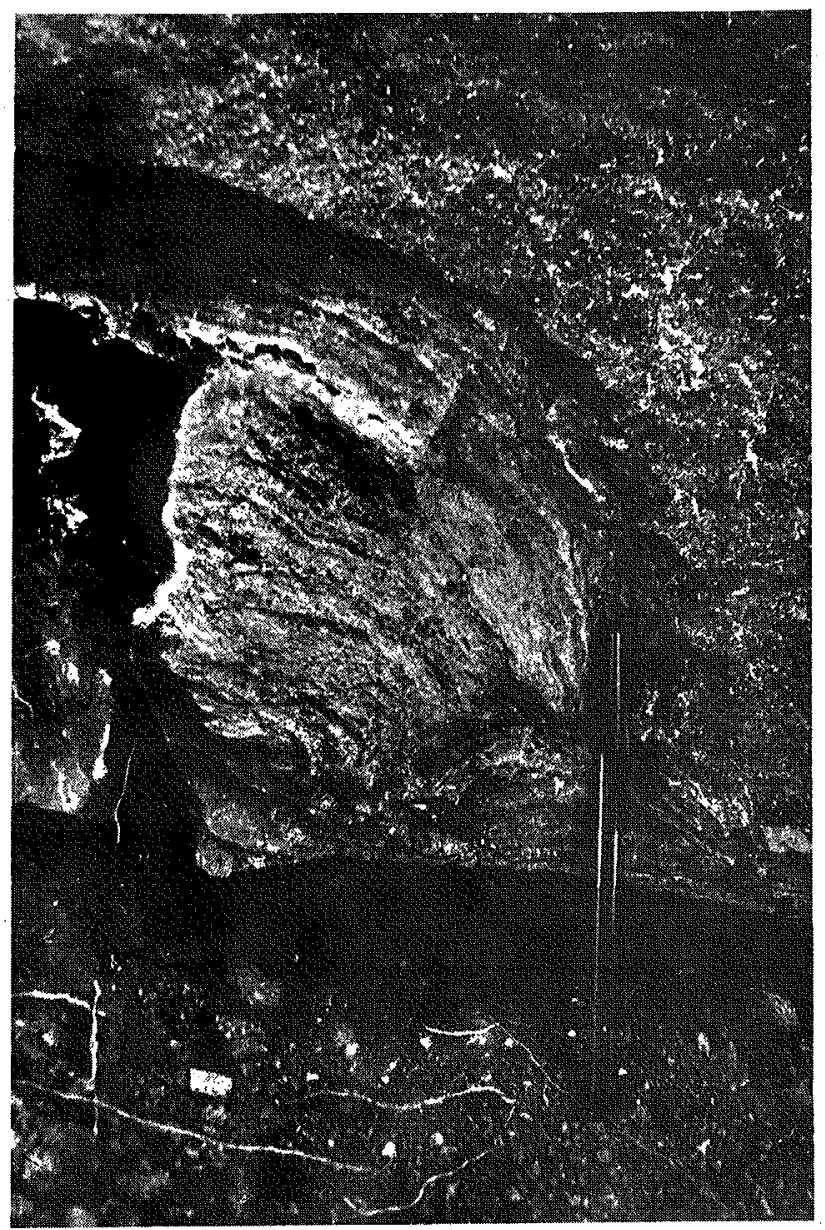

Photo 2 - Section verticale partielle d'un stromatolite. Paroi dûe à l'exploitation. Carrière principale

Les corps sont parfois tangents, mais le plus souvent séparés par quelques centimètres ou décimètres, rarement très isolés. Leur hauteur est généralement inférieure à $0,3 \mathrm{~m}$, en moyenne de l'ordre de 0,1 à $0,15 \mathrm{~m}$. Exceptionnellement ont été observés quelques individus doubles sur une même semelle ou formant deux bourgeons qui se ressoudent après avoir englobé le ciment. 


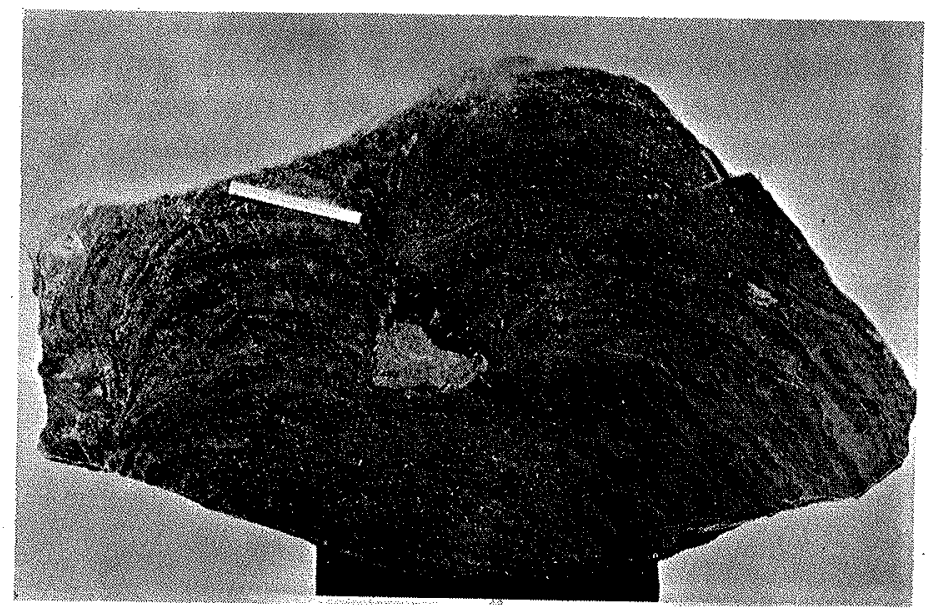

Photo 3 - Section exceptionnelle d'un stromatolite se bifidant à sa partie supérieure (Inzeria ?). Carrière principale. L'échelle est de $3 \mathrm{~cm}$

Le contact stromatolite-roche encaissante est toujours très net, quelquefois creusé par l'érosion.

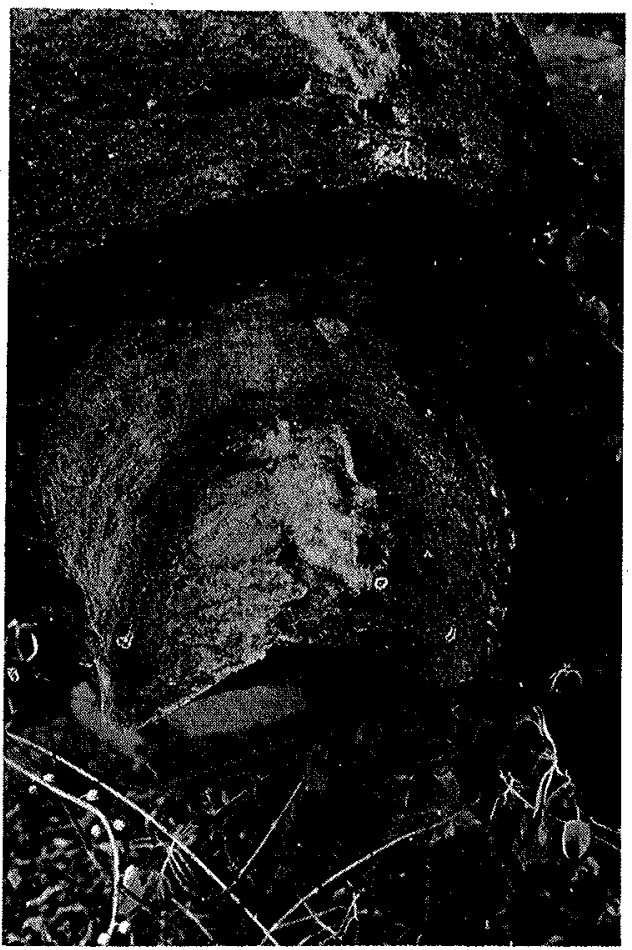

Photo 4 - Stromatolite obliquemment dégagé par l'érosion et détérioré par l'exploitation. Sommet de la carrière principale. Le diamètre de l'individu est de $23 \mathrm{~cm}$ 
Sections horizontales Fréquentes au sommet de la grande carrière, elles ont une forme circulaire ornée de légers festons. Le diamètre moyen est de l'ordre de $0,2 \mathrm{~m}$, le plus grand individu mesuré atteignant $0,45 \mathrm{~m}$. Les structures inférieures à $0,1 \mathrm{~m}$ sont peu communes. L'érosion différentielle met en relief les lamelles et les fragments de roche inclus dans le calcaire encaissant.

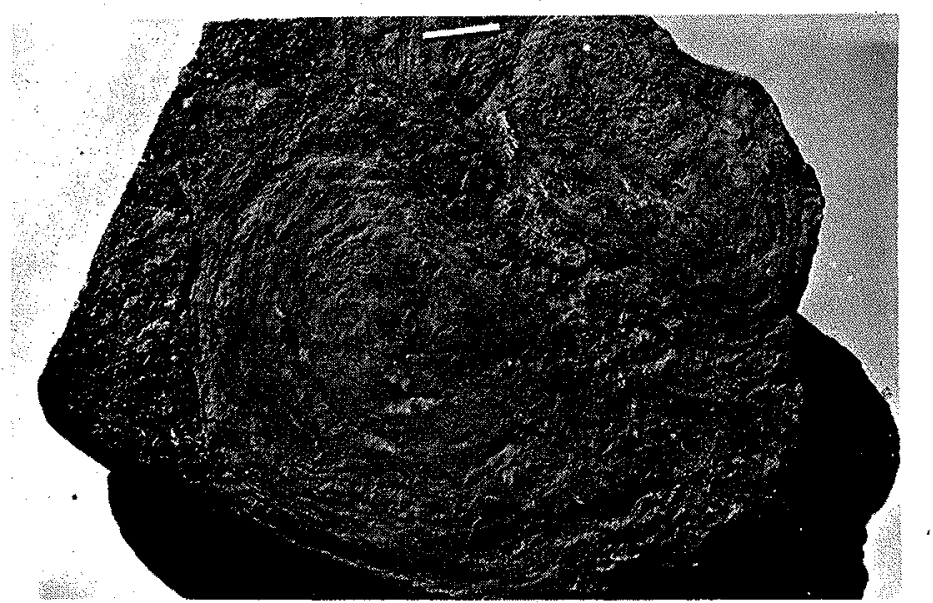

Photo 5 - Section horizontale naturelle de deux stromatolites sur une plaque de calcaire. Echantillon provenant du sommet de la carrière principale. L'échelle est de $3 \mathrm{~cm}$

2 - ETUDE MICROSCOPIQUE Lamelles L'ensemble, recristallisé en mosaïque, montre une alternance de bandes ondulées, claires et grossières ou plus foncées, plus fines et plus compactes que les précédentes, l'épaisseur des lits étant très variable. Des grains de quartz et de nombreux petits cristaux de pyrite, en agrégats ou isolés y sont irrégulièrement disséminés. Le contact entre les bandes est assez net et se fait par un passage très rapide.

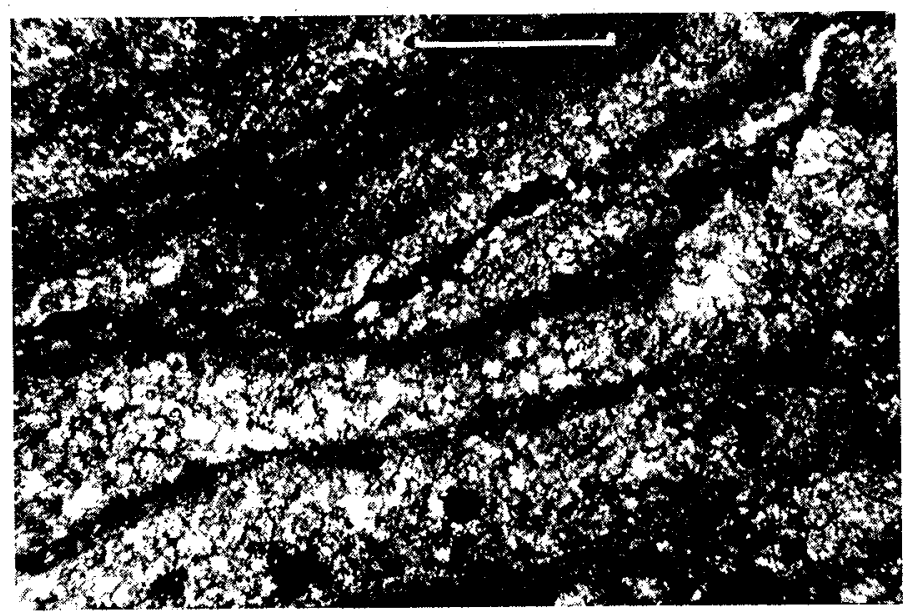

Photo 6 - Microstructure de stromatolite : alternance de bandes claires et foncées irrégulières. Lame mince, lumière naturelle. Le trait correspond à $0,25 \mathrm{~mm}$.

Observation: Cette lame mince comme les suivantes a une épaisseur supérieure á la normale afin de faciliter l'étude des structures organiques (Cf. Preiss, 1976 a) 
Les bandes claires sont formées de calcite en larges éléments avec très peu d'inclusions et quelques grains de quartz. On y observe localement des oolithes, en partie effacées par la diagenèse et des débris de roche. L'ensemble est identique au calcaire encaissant qui sera décrit ultérieurement.

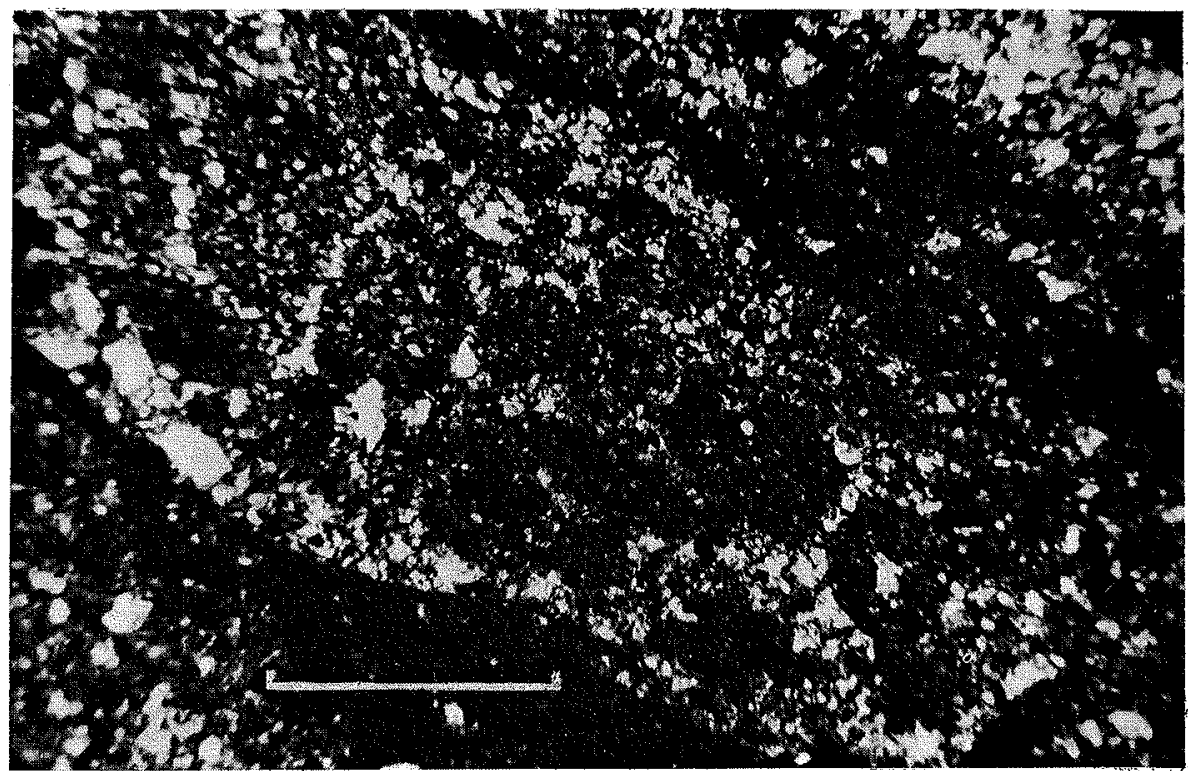

Photo 7 - Oolithes en partie effacées par la diagenèse, conservées dans une lamelle claire de stromatolite. Lame mince. Lumière naturelle. Le trait correspond à $0,25 \mathrm{~mm}$

Dans les cristaux le calcite des bandes foncées, d'innombrables petites inclusions sont visibles à fort grossissement (argile, limonite et/ou matiére organique). Certains lits présentent une structure longitudinale finement rubannée, d'autres un mince liseré beaucoup plus sombre, en haut ou en bas de la couche ou de part et d'autre de celle-ci. Les oolithes incluses sont très rares. Exceptionnellement des bandes foncées sont remplacées par une série de ponctuations irrégulières ou de courts filaments ondulés, s'anastomosant, l'ensemble dessinant des bandes subparallèles dans la couche plus claire. Il s'agit sans doute d'une fixation préférentielle d'impuretés par des micro-organismes.

Des lits opaques suivent localement les bandes (Streaky structure de Walter, 1972), ou les coupent en sifflet très aigus. Les veines de calcite transversales sont peu fréquentes.

Les lamelles ne sont pas toujours parallèles, s'anastomosant souvent, ou quelquefois se bifidant. Certaines sont tranchées en biseau et recouvertes obliquement par une nouvelle série attestant une érosion pendant le dépôt. Aucun gradedbedding n'a été observé.

Finalement, la recristallisation ne laisse quelquefois subsister que des alternances aux contours plus ou moins estompés. La chlorite, en longues aiguilles, est toujours rare.

En conclusion, si l'on adopte la classification de Bertrand-Sarfati (1976), les stromatolites de Serra Virgem ont ainsi une "microstructure simple du type film". 


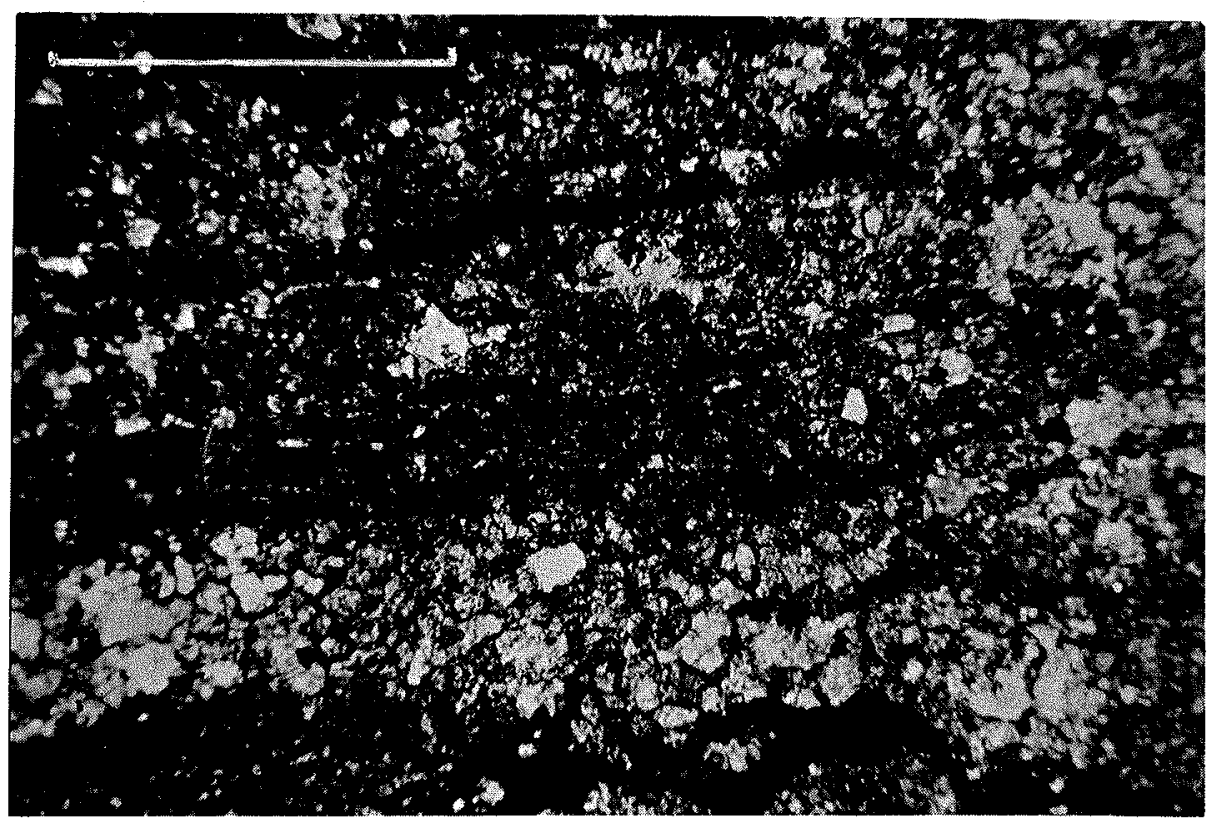

Photo 8 - Microstructure lamellaire feuilletée exceptionnelle, à l'intérieur d'une bande sombre. Lame mince. Lumière naturelle. Le trait correspond à $0,25 \mathrm{~mm}$

Contact avec la roche encaissante La limite, surtout lorsqu'elle se fait par une bande foncée, est toujours bien nette. Elle peut ou non être soulignée par un filonnet d'impuretés opaques ou par une veinule de calcite largement cristallisée.

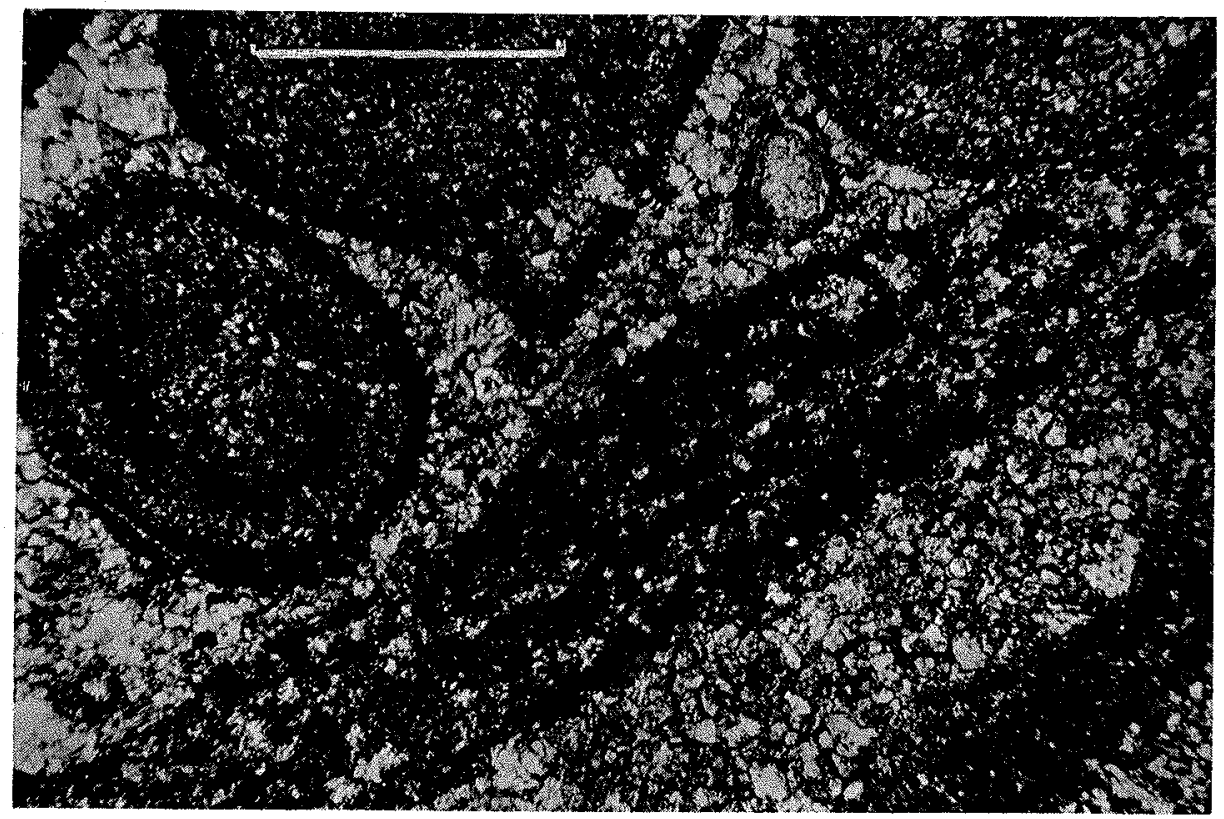

Photo 9 - Contact stromatolite-roche encaissante (moitié NW de la photo). Lame mince.

Lumière naturelle. Le trait correspond à $0,25 \mathrm{~mm}$ 
Parfois des oolithes microsparitiques, mais toujours en couches concentriques, viennent au contact du fossile et sont partiellement englobées dans sa périphérie, qui ne présente plus ainsi qu'une limite diffuse avec la roche encaissante. Ce cas se rapporte toujours aux parties des stromatolites enveloppées par une couche claire.

3 - DIAGNOSE DES STROMATOLITES Elle sera présentée dans le cadre défini par Bertrand-Sarfati (1972), à partir de travaux antérieurs.

3.1 - Morphologie des individus

Attitude: c'est la position du stromatolite dans le biostrome et par rapport à la sédimentation considérée comme horizontale. Les individus sont verticaux, leurs axes, courts, étant parallèles.

Volume: c'est le facteur de croissance. Les individus sont bulbeux.

Section: c'est la section horizontale des stromatolites. Il a été vu précédemment qu'elle est toujours subcirculaire,

Allure: c'est le rapport de la hauteur au diamètre. Sa faible valeur correspond à un stromatolite globuleux.

Bordure: c:est la surface latérale de la colonne. On a déjà vu qu'elle est lissse.

Convexité: c'est le profil de la lamination. Au sommet des individus où il est maximum, leur base étant subplate, le rapport hauteur du segment sur corde est de l'ordre de $1 / 3$, atteignant très rarement $1 / 2$.

Développement: c'est le volume occupé par les colonnes dans le biostrome, il est de type restreint, très ouvert, lès individus étant disséminés dans le biostrome.

Ramification: elle est nulle.

3.2 - Mode de groupement des individus

Le gîte fossilifère de Serra Virgem correspond à un biostrome.

3.3 - Microstructure

Simple, elle a été décrite en $\S^{2}$; les couches sombres sont en tapis homogène. 3.4 - Microfaciès de la roche encaissante

C'est un calcaire oolithique à débris de roche sédimenté durant la croissance du stromatolite, qui en inclut des oolithes dans ses couches claires.

Au microscope, les grandes oolithes sont : en minces couches microsparitiques aux tonalités alternantes, en lits clairs sparitiques séparés par de minces bảndes foncées plus fines, en noyaux monocristallins ou très finement recristallisées. Certaines ont un noyau de quartz, d'autres sont composées ou corrodées. Localement, enfin, toutes les oolithes ont recristallisé suivant la même direction comme le montre l'orientation des plans de macle.

Le ciment, sparitique, renferme des oolithes beaucoup plus petites et des gravelles claires entre lesquelles sont irrégulièremẹnt dispersés de grands fragments de calcaire et de stromatolites en lames allongées, aux angles peu arrondis (galettes), quelquefois êclatés. De grandes oolithes, simples ou multiples sont également déchiquetées, témoignant d'une diagenèse précoce mais incomplète. Des grains de quartz subanguleux sont assez fréquents dans le ciment.

Des veinules de calcite plus ou moins largement cristallisée recoupent l'ensemble. Elles sont bordées, au contact du ciment, par une frange d'éléments grossiers.

En quelques points, enfin, la roche est envahie par de grands cristaux de calcite, noyant dans un premier stade les oolithes dans de grandes plages claires cristallines, avant d'en effacer plus ou moins complètement la structure dans un stade plus avancé. 


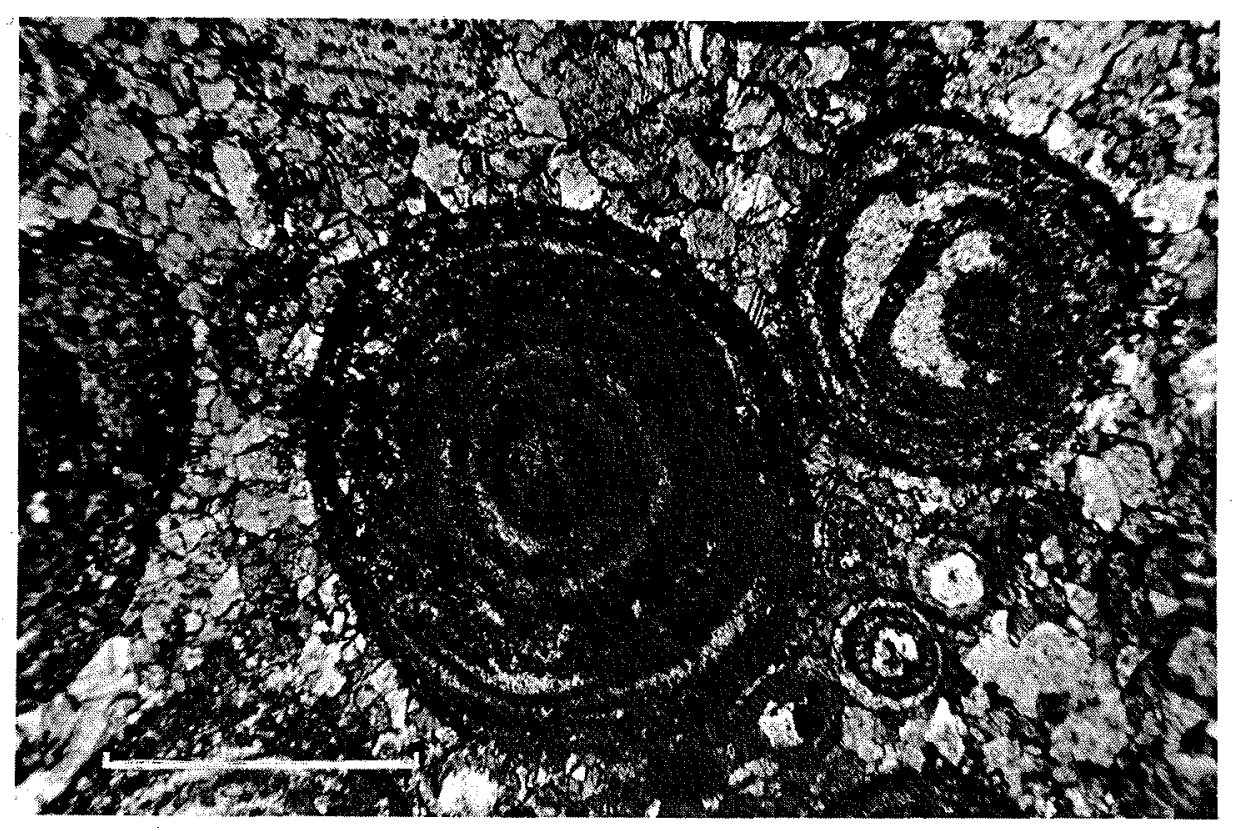

Photo 10 - Calcaire encaissant. De grandes oolithes sont noyées dans un ciment sparitique où des oolithes plus petites sont visibles. Lame mince. Lumière naturelle. Le trait correspond à $0,25 \mathrm{~mm}$

En conclusion: aucun ensemble de coupes sériées ne sera présenté en raison de la simplicité des structures organiques; le stromatolite est un coussin plus ou moins élevé, aux bords quelquefois redressés.

Classification et age Les stromatolites de Serra Virgem sont des Collenias (Krylov, 1976) du type globuleux ou noduleux de Koroljuk (1960), ou du sous-type globuleux de Krylov (in Hofmann, 1969). Pour Walter (1972) ils appartiennent au type "cumulate separate stromatolites".

Des formes analogues ont été étudiées dans le Riphéen du Nord de la Sibérie, mais la possibilité de les utiliser pour des corrélations à grande distance n'a pas encore été examinée (Raaben, 1969). Cette constatation ressort également du travail de Cloud et Semikhatov (1969).

Des "cumulate stromatolites" sont signalés par Walter (1972) à divers niveaux du Précambrien australien dans des roches dont les âges s'étagent de $2.020 \pm 165$ m.a. à 790 m.a. Aucune description détaillée n'en est donnée.

Selon Semikhatov (1976) des formes globuleuses (Colleniella et Paniscollenia décrites par Koroljuk en 1960) sont connues dans des terrains plus récents que 675 m.a. en Sibérie. Des formes plus anciennes (Nucleella décrite par Komar en 1966) se trouvent dans des roches dont l'âge est compris entre 1.500 e 675 m.a.

Aucune description détaillée accessible, pour ce type de forme simple, ne permet de réaliser un parallélisme avec les structures organiques de Serra Virgem, sauf peut être l'unique échantillon reproduit sur la photographie 3 et qui serait une forme atrophiée d'Inzeria. Ce groupe caractérise la base du Riphéen Supérieur (1.000. 
675 m.a. selon Preiss, $197.6 \mathrm{~b}$ ). On serait donc en présence de formes plus récentes que les Conophyton découverts par Moeri (1972) dans la région de Cabeludos (Minas Gerais) et datés de $1.650-950 \mathrm{~m} . \mathrm{a}$.

Cette conclusion, étayée par la position stratigraphique du faciès Lagoa do Jacaré, au-dessus de celui de Sete Lagoas, permet de supposer que les fossiles de Serra Virgem appartiennent à une autre séquence stromatolitique, plus récente, caractérisée par l'absence de conophyton et une sédimentation plus agitée. La même succession a été observée au Sahara Nord-Occidental par Bertrand-Sarfati (1972).

Analyses chimiques Les analyses chimiques d'un stromatolite (lamelles claires et foncées) et de sa roche encaissante, provenant du sommet de la carrière principale, sonit les suivantes:

\begin{tabular}{lcc} 
& Stromatolite & Roche encaissante \\
Perte au feu & 37,02 & 40,47 \\
$\mathrm{CaO}$ & 47,77 & 53,49 \\
$\mathrm{MgO}$ & 0,27 & 0,17 \\
$\mathrm{R}_{2} \mathrm{O}_{3}$ & 1,16 & 0,52 \\
$\mathrm{Résidu} \mathrm{insoluble}_{\mathrm{P}_{2} \mathrm{O}_{5}}$ & 12,59 & 5,24 \\
& 0,09 & 0,08 \\
\cline { 2 - 3 } & $\mathbf{9 8 , 9 0}$ & 99,97
\end{tabular}

(Analyste: J.O. Cassedanne)

Comme dans les autres gîtes fossilifères du même groupe, les structures organiques diffèrent chimiquement de la roche encaissante : elles sont plus riches en matières insolubles (argile essentiellement et un peu de quartz) et un peu plus riches en, oxydes (pyrite dosée comme $\mathrm{Fe}_{2} \mathrm{O}_{3}$ ). En raison de la faible quantité de magnésium présente, il n'est pas permis d'observer de différence notable entre les teneurs de cet élément dans le fossile et dans la roche encaissante, contrairement à ce qui se produit pour les stromatolites d'autres gisements du même Groupe, tant à Bahia qu'à Minas Gerais (Cassedanne, 1964 \& 1968 a) ou du Supergroupe Minas (Cassedanne et Cassedanne, 1976), où la roche encaissante est plus dolomitique.

L'absence de phosphore est également notable, contraire aux faits observês dans les autres gisements.

Ambiance de sedimentation La séquence lithologique de la colline de Serra Virgem démontre une sédimentation calcaire prédominante, en eaux peu. profondes, chaudes et agitées, se traduisant par d'épais lits oolithiques. Ce type de roche est également bien représenté dans la vallée du Rio São Francisco (Cassedanne, 1968 b), mais appartient plus généralement au faciès Sete Lagoas.

Les fragments de calcaire subanguleux, précocement indurés et la fréquence des débris allongés de stromatolites dans le ciment encaissant les organismes, attestent des remous locaux importants se traduisant parfois par l'abrasion locale de certaines lamelles, mais aussi l'absence de transport à longue distance. Les stromatolites, formant un biostrome peu dense, ont été ensevelis, en partie par les produits de leur destruction, dans une mer agitée, peut être par des courants de marée circulant dans des passes peu profondes. 
Des structures morphologiquement voisines de celles de Serra Virgem sont mentionnées par Donaldson (1976) dans les Territoires du NW Canadien, également associées à des ripple-marks, stratification entrecroisée, oolithes, fentes de dessication et conglomérat intraformationnel. Cet auteur considère que ces organismes ont également vécu dans un milieu de turbulence élevée, situé dans la zone intertidale ou quelquefois supratidale.

De légers mouvements locaux d'abaissement ou de surélévation du bassin sédimentaire sont responsables des variations pétrographiques de la série lithologique : horizons de phyllites intercalés dans une séquence carbonatée et variations des microfaciès des divers calcaires oolithiques. En outre ces mouvements se sont traduits sporadiquement par des émersions qu'attestent fentes de dessication et galets de boue. Les grains de quartz, subanguleux, fréquents, proviendraient d'une terre émergée voisine.

On retrouve donc des conditions de sédimentation déjà mises en évidence dans d'autres gites fossilifères du même Groupe (Cassedanne, 1968 c) : dans une anse ou une baie peu profonde, au fond agité de légers mouvements sporadiques, et aux eaux agitées, se sont formés des dépôts calcaires (oolithiques) entourant des organismes de même composition. Ces deux derniers points diffèrent des faits observés dans les autres gisements où les roches sont plus gênéralement dolomitiques (comme les organismes), sans oolithes.

Il est possible que cette différence de milieu, succédant à des dépôts dolomitiques à Conophyton (abstraction faite des siltites) soit responsable de la forme des stromatolites de Serra Virgem, différents de ceux décrits jusqu'à ce jour au Brésil.

CONCLUSION Les stromatolites globuleux de Serra Virgem appartiennent à un ensemble lithologique calcaire riche en oolithes, vraisemblablement plus récent qué celui renfermaint les autres structures précédemment décrites dans dés formations surtout dolomitiques.

\section{BIBLIOGRAPHIE}

BARBOSA O. - 1965 - Série Bambuí. Simpósio sobre as formações Eo-Paleozóicas do Brasil. Rio de Janeiro XIX: Congr. S.B.G.

BERTRAND-SARFATI J. - 1972 - Stromatolites columnaires du Précambrien Supérieur du Sahara Nord-Occidental. Inventaire, morphologie et microstructure des laminations. Corrélations stratigraphiques. Paris C.N.R.S. Ed. C.R.Z.A. Sér. Géol. n. 14 $245 \mathrm{pp}$.

BERTRAND-SARFATI J. - 1976 - An attempt to classify late Precambrian stromatolite microstructures. Amsterdam Elsevier Ed. Chap. 5.2 in "Stromatolites" Develop. in Sedimentology n:20 Ed. by M.R. Walter 790 pp.

BRANCO J.J.R. \& COSTA M.T. da - 1961 - Roteiro para a excursão Belo Horizonte Brasília. Belo Horizonte I.P.R. Publ. n?15 Contr. XIV! Congr. S.B.G.

BRAUN O.P.G. - 1968 - Contribuição à estratigrafia do Grupo Bambuí. Anais do XXII Congr. Soc. Bras. Geol., Belo. Horizonte, pp. 155-166.

CASSEDANNE J.P. - 1964 - Biostrome à Collenia dans le Calcaire de Bambui. Anais Acad. Bras. Ciências 36 n: 1 p. 49-58.

CASSEDANNE J.P. - 1966 - Niveau à galets mous dans le calcaire de Bambui. Anais Acad. Bras. Ciências 38 (2) : 281-288.

CASSEDANNE J.P. - 1968a - Description du biostrome à Collenias de la Mine de Vazante (Minas Gerais). Anais Acad. Bras. Ciências 40 (2): 215-225. 
CASSEDANNE J.P. - 1968b - Contribution à l'étude des calcaires de Bambui : Microfaciès et analyses des formations carbonatées encaissant les gîtes de plomb et de zinc brêsiliens. Inst. Geoc. U.F.R.J. Bol. Geol. n? 2 p. 38-62.

CASSEDANNE J.P. - 1968c - Nota sobre o ambiente de sedimentação das rochas encaixando a mineralização de Vazante (Minas Gerais). Anais XXII. Congr. Soc. Bras. Geol. p. 33:40.

CASSEDANNE J.P. \& CASSEDANNE J.O. - 1976 - Les stromatolites columnaires de la carrière du Cumbe (Série Minas - Brésil). Bull. Soc. géol. France 7\% Série Tome XVIII (4):959-965.

CLOUD P.E. \& SEMIKHATOV M.A. - 1969 - Proterozoic stromatolite zonation. Am. Journ. Sciences (267):1017-1061.

DONALDSON J.A. - 1976 - Palaecology of Conophyton and associated stromatolites in the Precambrian Dismal Lake and Rae Group, Canada. Chap. 10.2 in "Stromatolites", Developments in Sedimentology.

HOFMANN H.J. - 1969 - Attributes of stromatolites. Ottawa Geol. Survey of Canada Paper 69-39 43 pp.

KOROLJUK I.K. - 1960 - Subdivision of the Cambrian and pre-Cambrian of Eastern Siberia according to stromatolites. Copenhagen Rep. XXI Session Norden Int. Geol. Congress, Part VII, p. 113-118.

KRYLOV I.N. - 1976 - Approaches to the classification of stromatolites. Chap. 2.4 in "Stromatolites". Developments in Sedimentology.

MARCHESE M.G. - 1974 - Estromatolitos "gymnosolenidos" en el lado oriental de Minas Gerais. Brasil. Rev. Bras. Geoc. 4(4):257-261.

MOERI E. - 1972 - On a columnar stromatolite in the Precambrian Bambui Group of Central Brazil. Ecl. Geol. Helv. 65(1):185-195.

PREISS W.V. - 1976 a - Basic field and laboratory methods for the study of stromatolites. Chap. 2.1 in "Stromatolites" Developments in Sedimentology.

PREISS W.V. - 1976 b - Intercontinental correlation. Chap. 7.2 in "Stromatolites" Developments in Sedimentology.

RAABEN M.E. - 1969 - Columnar stromatolites and late Precambrian stratigraphy. Am. Journ. Sciences (267):1-18.

SEMIKHATOV M.A. - 1976 - Experience in stromatolite studies in the U.S.S.R. Chap. 7.1 in "Stromatolites" Developments in Sedimentology.

WALTER M.R. - 1972 - Stromatolites and the biostratigraphy of the australian Precambrian and Cambrian. The Palaeontological Assoc. London, Sp. Paper in $\mathrm{Pa}$ laeontology n:11, 190 pp. 